\title{
Non associative damage interface model for mixed mode delamination and frictional contact
}

\author{
F. Parrinello ${ }^{\mathrm{a}}$, G. Borino ${ }^{\mathrm{a}}$ \\ ${ }^{a}$ Dipartimento di Ingegneria, University of Palermo; Viale delle Scienze, 90128 Palermo, \\ Italy
}

\begin{abstract}
The present paper proposes a new interface constitutive model based on the nonassociative damage mechanics and frictional plasticity. The model is developed in a thermodynamically consistent framework, with three independent damage variables. The non associative flow rules drive the concurrent evolution of the three damage variables. The interface model provides two independent values for the mode I fracture energy and the mode II fracture energy and it is able to accurately reproduce arbitrary mixed mode fracture conditions. The model can also take into account the presence of frictional effects both at the fully debonded zones and at the partially debonded ones. The experimental tests developed by Benzeggagh and Kenane with seven different mixed mode ratios have been numerically simulated with a unique set of constitutive parameters. The split shear torsion, for the evaluation of the mode III delamination toughness, has been analysed by a three-dimensional numerical simulation.
\end{abstract}

Keywords: Damage, Delamination, Fracture, Mixed mode, Interface model, Friction.

\section{Introduction}

In the last decade, Cohesive Zone Models (CZMs) have assumed a considerable rule for the analysis of fracture mechanic problems for its capability to reproduce fracture and delamination phenomena in the framework of rational constitutive modelling. Since the pioneering work of Dugdale and Barenblatt $[11,3]$ CZMs represent a powerful alternative to the linear elastic fracture mechanics theory. Moreover, CZMs have a relatively simple numerical implementation by means of interface models, which accurately reproduces fracture and delamination processes, provided that proper cohesive laws are established.

Recently, special attention has been focused on the assessment of the consistency of existing interface constitutive models under mixed mode delamination

\footnotetext{
* Corresponding author

Email address: francesco.parrinello@unipa.it (F. Parrinello)
} 
condition, especially from the thermodynamics point of view. In Dimitri et al [10] four constitutive models are analysed in order to evaluate their response under mixed mode debonding conditions and whether they are consistent in terms of stress and energy dissipation. In [10] it is shown that the CZMs proposed in $[21,16,6]$ may produce physically inconsistent results, whereas responses of the model proposed by van den Bosch [36] results to be coherent with experimental evidences. Conversely, such model is not based on a potential function and it is not developed in order to comply with thermodynamics principles. Moreover, in [36] an unloading law, different than the loading one, is not explicitly defined and energy dissipation cannot be evaluated. In that model two independent laws are defined, respectively for the tangential traction component and for the normal one and, as already stated in [23], symmetry requirement of the tangent stiffness matrix is not satisfied. Recently, a new promising framework for the formulation of interface cohesive models for mixed mode delamination with variable mode-ratio has been presented in [8].

In [10] a thermodynamically consistent model, defined as evolution of the van den Bosch et al model [36], but based on a Helmholtz free energy functional has been proposed. The same form of the tangential and normal traction components proposed in [36] are rigorously derived by applying the Coleman and Noll [7] procedure through definition of four independent scalar damage variables.

In [21] potential-based models and non-potential-based models are analysed for mixed mode separation loading paths and under over-closure conditions. The non-physical behaviour of the Xu-Needleman model [37] emerges, with negative dissipation or repulsive normal traction in mixed mode delamination conditions, when fracture energy in mode II is assumed greater than fracture energy in mode I. The behaviour of some traction separation laws under mixed mode delamination condition is analysed also in [34, 24].

In [33] a thermodynamically consistent cohesive frictional model with different mode I and mode II fracture energies is presented. The model is defined by a single scalar damage variable and the authors argue that the dissipation performed by the modelled damaging phenomenon, which is equal to the separation work, in pure mode I $\left(G_{I}\right)$ and in pure mode II $\left(G_{I I}\right)$ are coincident, that is $G_{I}=G_{I I}$. Moreover, the authors ascribe only to the frictional effects the greater value of mode II fracture energy $G_{I I}$ with respect to $G_{I}$, experimentally observed. Such effects are increased by considering, at the mesoscale level, the geometry of the interface in the form of a periodic arrangement of distinct inclined planes. The thermodynamic consistency of the well known potential based model proposed by Park, Paulino and Roesler in [25] is investigated in [34].

The contribution of frictional behaviour to the the mode II dissipation energy has been analysed under increasing cycling load in [32] by the cohesive-frictional interface model proposed in $[29,30]$ for small displacements and in [28] for large displacement analysis. An improved model has been presented in [31] with two independent values of the fracture energies under pure mode I and pure mode II delamination conditions. Such a model is defined by a damage activation 
condition which depend on the separation displacement components. The analytical solution of the mode II fracture energy in the 4ENF test with frictional effects has been proposed in [27]. The frictional behaviour has been modelled in [13] through an elastic-plastic interface model in a multi-scale computational strategy for the analysis of masonry structures. In [35] the frictional tractions are coupled to the cohesive tractions of the potential based model proposed in [25] for the homogenization of the debonding of the particle reinforced composites, and in [2] the coupled cohesive frictional-contact model is integrated for the prediction of the dynamic fracture under compression.

The models listed above are just a largely incomplete description of the huge amount of contributions describing interface constitutive relations. The interested readers can refer to $[8,25,33]$ for an in-depth review of contributions on the subject. Recently, the general trend in modelling interface laws is the development of such relations in the proper constitutive framework. Initially, simple traction-separation displacement relations were proposed. Then, it was considered rational to define these relations by introducing a potential function and, more recently, by considering the very dissipative nature of the cohesive interface with path dependent response. A specific attention has been posed to the thermodynamic consistency of the interface constitutive relations, especially for model with different values of the fracture energy in mode I, mode II and mixed mode debonding conditions. In the present paper a new thermodynamically consistent CZM is developed in the framework of non-associative damage mechanics with one scalar damage variable for each displacement jump component. It is based on a Helmholtz free energy, which play the role of potential not only for the traction components but also for the three damage variables and for the other internal variables.

The proposed model cannot be defined, in general terms, superior to the multitude of available models, even if it can reproduce quite accurately many experimental tests, but rather it gives a new light to the theoretical framework of the interface constitutive models. In fact, it allows to model independent damage activation conditions for some different debonding conditions (mode I, mode II or mixed mode) and the non-associative flow rule drives the concurrent evolution of the three damage variables and the concurrent degradation of all the traction components.

The presented model produces independent works of separation in pure mode I and pure mode II delamination conditions and it can also take into account the frictional effects with a smooth transition, from the initial elastic behaviour of the sound material, up to the fully debonded behaviour with frictional residual strength. The cohesive-frictional behaviour is based on the same mesoscale interpretation of the scalar damage variable, previously proposed in $[29,1]$. Traction components, damage evolution and the relevant constitutive equations are derived by following the classical Coleman and Noll procedure [7] and the model satisfies the second thermodynamic law by proving that dissipation is non-negative for any loading path. 


\section{The non-associative damage model model}

Let us consider two solids occupying the bounded domains $\Omega^{+}$and $\Omega^{-}$. The two bodies are mutually bonded or in cohesive contact on the common surface $\Gamma$. On the overall system $\Omega=\Omega^{+} \cup \Omega^{-} \cup \Gamma \subset \mathbb{R}^{3}$ are applied external tractions $\boldsymbol{t}$ on the free boundary $\Gamma_{t}$ and imposed displacement $\overline{\boldsymbol{u}}$ on the constrained boundary surface $\Gamma_{u}$, as represented in Fig.1a. The hypotheses of homogeneous material and small strain are assumed. The interface constitutive model is developed with reference to a zero thickness interface, whose kinematic behaviour is represented in Fig. 1b by the separation displacement between the two bonded solid domains. The interface constitutive model is defined in a local reference frame with axes $\boldsymbol{e}_{1}$ and $\boldsymbol{e}_{2}$ tangent to the surface $\Gamma$ and normal axis $\boldsymbol{e}_{3} \equiv \boldsymbol{n}$. The displacement jump across the interface is defined by means of the kinematic variable $\llbracket \boldsymbol{u} \rrbracket=\boldsymbol{u}^{+}-\boldsymbol{u}^{-}$, where $\boldsymbol{u}^{+}$and $\boldsymbol{u}^{-}$are the displacement vectors, respectively, on the positive side and on the negative side of the internal surface $\Gamma$, as shown in Fig. 1b. The positive side $\Gamma^{+}$is defined with respect to the orientation of the outward normal vector $\boldsymbol{e}_{3} \equiv \boldsymbol{n}$ to the interface surface.

In interface formulation, the displacement jump is a strain measure and it can be decomposed in the two tangential components $u_{1}=\llbracket \boldsymbol{u} \rrbracket \cdot \boldsymbol{e}_{1}$ and $u_{2}=\llbracket \boldsymbol{u} \rrbracket \cdot \boldsymbol{e}_{2}$, and the normal component $u_{n} \equiv u_{3}=\llbracket \boldsymbol{u} \rrbracket \cdot \boldsymbol{e}_{3}$, with reference to the interface surface.

The interface equilibrium condition is represented by the following relation $\llbracket \mathbf{t} \rrbracket=\mathbf{t}^{+}-\mathbf{t}^{-}=0$, where $\mathbf{t}^{+}$and $\mathbf{t}^{-}$are the traction vectors, respectively, on the positive side and on the negative one, as represented in Fig. 1b; for sake of simplicity tractions are defined as $\mathbf{t}:=\mathbf{t}^{+}=-\mathbf{t}^{-}$and displacement jumps are defined as $\boldsymbol{u}:=\llbracket \boldsymbol{u} \rrbracket$.

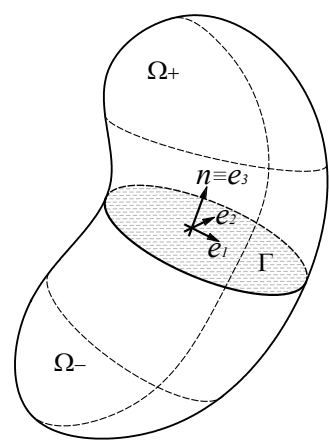

a)

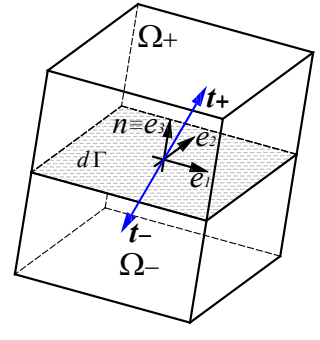

b)

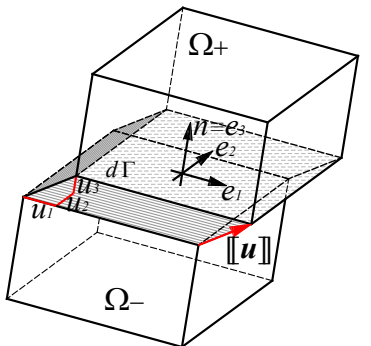

c)

Figure 1: a) The two solids bonded on the common internal surface $\Gamma$. b) An element of the internal surface $\Gamma$ with the local reference frame axes $\boldsymbol{e}_{1}, \boldsymbol{e}_{2}, \boldsymbol{e}_{3} \equiv \boldsymbol{n}$ and representation of the tractions $\mathbf{t}^{+}$on surface $\Gamma^{+}$and $\mathbf{t}^{-}$on surface $\Gamma^{-}$. c) The components of the separation displacement are represented on the deformed configuration of the RSE.

In the damage mechanics framework the Representative Surface Element (RSE) of interface, whose size is assumed small compared to the structural sizes and sufficiently large compared to the material inhomogeneities, is considered 
and the relevant average damage variable $\omega$ is generally defined (see [20]) as the ratio between the area $d A_{d}$ of the debonded fraction of RSE and the whole RSE area $d A$; that is

$$
\omega=\frac{d A_{d}}{d A}
$$

In Fig. 2 a partially cracked Representative Surface Element of the interface $\Gamma$, connecting two solid domains $\Omega^{+}$and $\Omega^{-}$, is depicted in both the reference and in the deformed configuration.

The interface constitutive model is developed in the same phenomenological framework proposed in [29,31], where the damage variable is also considered as a discriminant parameter which governs the transition form the initial elastic behaviour, of the sound interface, to the residual frictional behaviour, of the fully delaminated interface. The behaviour of the partially cracked interface is developed adopting a mixture rule, at the sub-scale where the two fractions are respectively: the cracked fraction with a frictional behaviour and traction $\mathbf{t}_{f}$, whose area is $\omega d \Gamma$; the sound fraction of RSE, whose area is $(1-\omega) d \Gamma$, with a cohesive elastic behaviour and traction $\mathbf{t}_{c}$. In the following, the index $c$ is used for the cohesive fraction variables and index $f$ for variables of the frictional portion.

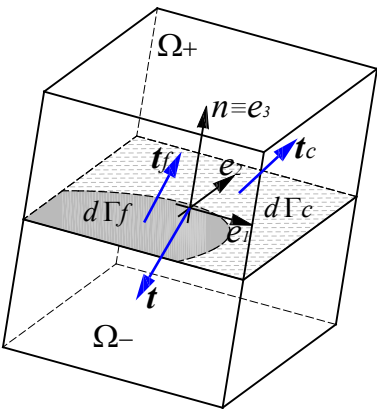

a)

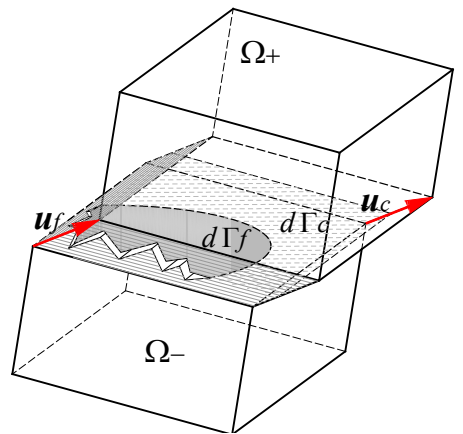

b)

Figure 2: Partially cracked Representative Surface Element of interface $\Gamma$

Since the present approach is based on a mixture theory with two fractions, it is allowed to define specific displacement jump vectors $\boldsymbol{u}_{f}$ and $\boldsymbol{u}_{c}$ for each fraction.

The behaviour in the cohesive (or sound) fraction is assumed as purely elastic and the relevant elastic deformation is measured by the following variable

$$
\delta^{c e}=\boldsymbol{u}_{c} .
$$

The cracked fraction is modelled as elastic-plastic with null strength for tensile normal traction. As pointed out in [29, 31], the elastic deformation of the frictional part can be regarded as the elastic deformation of asperities and it is defined as

$$
\boldsymbol{\delta}^{f e}=\boldsymbol{u}_{f}-\boldsymbol{\delta}^{f p}-\boldsymbol{\delta}^{f d} .
$$


where $\boldsymbol{\delta}^{f p}$ is its plastic component and $\boldsymbol{\delta}^{f d}$ is a detachment displacement (see [29] for more details). The interface deformation components of the cracked fraction $\omega d \Gamma$ of the representative surface element $d \Gamma$ are graphically represented in the Figures 3 a, d. The elastic deformation $\boldsymbol{\delta}^{f e}$ (Fig.3b) models the elastic and reversible deformation of asperities. The plastic deformation $\boldsymbol{\delta}^{f p}$ (Fig.3c) models the displacement jump caused by the irreversible sliding of asperities, which evolves when the limit frictional strength is attained, producing plastic dissipation energy. Detachment displacement $\boldsymbol{\delta}^{f d}$ (Fig.3d) is considered in order to model the opening separation displacement, with null traction in the cracked fraction and null energy dissipation.
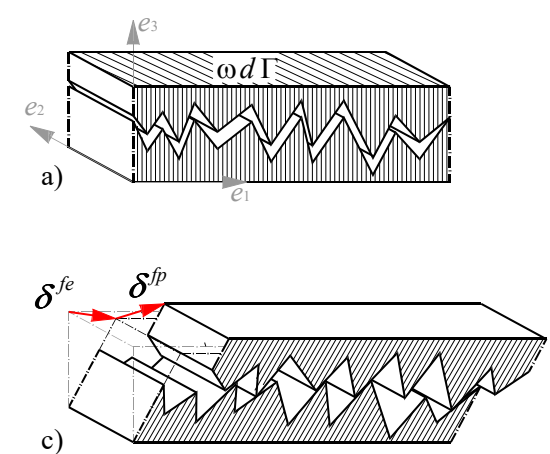
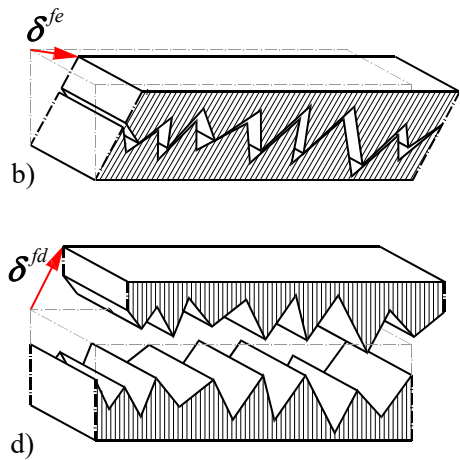

Figure 3: Representation of elastic, plastic and detachment deformation components of the cracked fraction $\omega d \Gamma$ of a Representative Surface Element of interface $\Gamma$

From a kinematical point of view, normal component of elastic displacement and normal detachment component cannot be active at the same time and the following complementarity relations hold

$$
\delta_{n}^{f e} \leq 0, \quad \delta_{n}^{f d} \geq 0, \quad \delta_{n}^{f e} \delta_{n}^{f d}=0, \quad \delta_{t}^{f e} \delta_{n}^{f d}=0 .
$$

The above relations state that the frictional elastic components of interface deformation $\left(\delta_{t}^{f e}\right.$ and $\left.\delta_{n}^{f e}\right)$ are null in case of full separation with positive normal detachment displacement $\left(\delta_{n}^{f d}>0\right)$. In the previous equations, state variables and configuration variables have been introduced and the discontinuity displacement vectors $\boldsymbol{u}_{c}$ and $\boldsymbol{u}_{f}$ define the deformed configurations of the two fractions. The two discontinuity displacement vectors are only formally distinguished one from another. In fact, as represented in Fig. 2b, the deformed configurations of the two fractions are not described independently from each other in the mesoscale constitutive modelling; on the contrary, the pointwise kinematic configuration is uniquely defined by means of the separation displacement $\boldsymbol{u}$ and the following internal compatibility condition is assumed

$$
\boldsymbol{u}=\boldsymbol{u}_{c}=\boldsymbol{u}_{f}
$$




\subsection{Thermodynamic framework}

The behaviour of interfaces depends on the separation mode, with different values of strength, in terms of maximum traction, and different values of fracture energy for the opening separation mode and for the sliding separation one. Such a phenomenon is well known in literature and several contributions have been proposed $[21,25,10,36,33]$ and a cohesive zone model with two independent fracture energy values in mode I and in mode II has been developed, in a consistent thermodynamic approach, by the same authors in [31], for a two-dimensional interface.

The interface constitutive model proposed in the present paper is developed in the rigorous thermodynamic framework of the non-associative damage mechanics. The model is able to produces independent fracture energy values in pure mode I and in pure mode II delamination mode and it also allows to model the residual frictional strength of the fully debonded interface and the transition from the initial elastic behaviour to the residual frictional one. The proposed interface constitutive model represents an alternative and original interpretation of the model proposed in [31], which is a two-dimensional constitutive model with a single scalar damage variable. On the contrary, the proposed formulation provides a three-dimensional constitutive model and it requires three independent damage variables $\omega_{i}$ with $i=1,2,3$, one for each traction component $t_{i}$. This choice can appear in contrast with the classic geometric interpretation of the interface damage variable defined in Eq.(1) (see [20]). Namely, a scalar variable which governs uniformly the degradation of the three components of the mutual tractions acting between the two connected surfaces. Moreover, at the full debonding condition $(\omega=1)$, and excluding possible frictional traction, all traction components have to be null and this condition can be particularly difficult to be verified for non scalar damage models. But the proposed nonassociative damage model intrinsically produces the same increment for all the damage variables, for any debonding condition.

The cohesive-frictional model is developed in a rigorous thermodynamic framework and it is based on the following Helmholtz free energy function

$$
\psi=W_{e l}^{c}+W_{e l}^{f}+\Psi_{i n}(\xi)
$$

where $W_{e l}^{c}$ is the elastic-damage strain energy of the cohesive fraction, $W_{e l}^{f}$ is the elastic-damage strain energy of the frictional fraction and $\Psi_{i n}(\xi)$ is the internal energy, function of the scalar kinematic internal variable $\xi$. The elastic strain energies of the two fractions are defined as

$$
\begin{aligned}
W_{e l}^{c} & =\sum_{i=1}^{3} \frac{1}{2}\left(1-\omega_{i}\right) K_{i}^{c} \delta_{i}^{c e 2} \\
W_{e l}^{f} & =\sum_{i=1}^{3} \frac{1}{2} \omega_{i} K_{i}^{f} \delta_{i}^{f e^{2}} .
\end{aligned}
$$

The elastic strain energy of the frictional fraction in Eq.(8) does not distinguish between compressive and tensile normal traction. The presence of frictional 
traction with tensile normal component is precluded by the kinematic complementary conditions of unilateral frictional contact in Eq.(4).

If the interface behaviour is assumed isotropic in the plane $\boldsymbol{e}_{1}, \boldsymbol{e}_{2}$ tangent to the surface $\Gamma$, the elastic stiffness, strength and fracture energy are equal in the two tangent direction $\boldsymbol{e}_{1}$ and $\boldsymbol{e}_{2}$ and therefore

- $K_{1}^{c}=K_{2}^{c}=K_{t}^{c}$ are the elastic tangent stiffness parameters of the cohesive fraction;

- $K_{3}^{c}=K_{n}^{c}$ is the elastic normal stiffness parameter of the cohesive fraction;

- $K_{1}^{f}=K_{2}^{f}=K_{t}^{f}$ are elastic tangent stiffness parameters of the frictional behaviour;

- $K_{3}^{f}=K_{n}^{f}$ is the elastic normal stiffness parameter of the frictional behaviour.

The constitutive parameters $K_{i}^{f}$ measure the elastic stiffness of the asperities of the cracked fraction and are therefore related to the residual frictional behaviour of the damaged interface, so the frictional elastic stiffnesses have to be smaller than the cohesive ones, at least for the tangential components, that is $K_{i}^{f}<K_{i}^{c}$ with $i=1,2$ and $K_{3}^{f} \leq K_{3}^{c}$ (see [29] for more details).

The proposed CZM can be framed in the class of intrinsic interface formulations, which model an initial elastic behaviour, and the elastic stiffness parameters $K_{i}^{c}$ and $K_{i}^{f}$ can be regarded as penalty parameters for the evaluation of the interface traction components before the damage activation condition is attained in the cohesive fraction, or the frictional sliding condition is attained in the relevant fraction. The development of the proposed CZM in the class of extrinsic formulations, with initial rigid behaviour to avoid the unphysical use of penalty terms, can be approached by the discontinuous Galerkin method, as proposed in $[15,22,14]$. Nevertheless, the development of the extrinsic formulation of the proposed model requires a further investigation, which is not deepened in the present paper.

In order to develop suitable evolution equations for damage and plasticity variables, thermodynamic consistency, in the form of the second principle, can be enforced by the Clausius-Duhem inequality, which gives an explicit form for the non-negative mechanical energy dissipation density:

$$
D=\boldsymbol{t} \cdot \dot{\boldsymbol{u}}-\dot{\psi} \geq 0
$$

The rate of Helmholtz free energy can be derived from Eqs.(6-8) as follows

$$
\begin{aligned}
\dot{\psi} & =\sum_{i=1}^{3}\left(\frac{\partial \psi}{\partial \delta_{i}^{c e}} \dot{\delta}_{i}^{c e}+\frac{\partial \psi}{\partial \delta_{i}^{f e}} \dot{\delta}_{i}^{f e}+\frac{\partial \psi}{\partial \omega_{i}} \dot{\omega}_{i}\right)+\frac{\partial \psi}{\partial \xi} \dot{\xi} \\
& =\sum_{i=1}^{3}\left(t_{i}^{c} \dot{\delta}_{i}^{c e}+t_{i}^{f} \dot{\delta}_{i}^{f e}-Y_{i} \dot{\omega}_{i}\right)-\chi \dot{\xi}
\end{aligned}
$$


where:

$$
\begin{aligned}
Y_{i} & :=-\frac{\partial \psi}{\partial \omega_{i}}=\frac{1}{2} K_{i}^{c} \delta_{i}^{c e 2}-\frac{1}{2} K_{1}^{f} \delta_{i}^{f e^{2}} \\
t_{i}^{c} & :=\frac{\partial \psi}{\partial \delta_{i}^{c e}}=\left(1-\omega_{i}\right) K_{i}^{c} \delta_{i}^{c e} \\
t_{i}^{f} & :=\frac{\partial \psi}{\partial \delta_{i}^{f e}}=\omega_{i} K_{i}^{f} \delta_{i}^{f e} \\
\chi(\xi) & :=-\frac{\partial \psi_{i n}}{\partial \xi} .
\end{aligned}
$$

In Eqs.(11)-(14) $Y_{i}$ are the energy release rates, that are the conjugated variables of damage variables $\omega_{i} ; t_{i}^{c}$ and $t_{i}^{f}$ are the cohesive traction and the frictional traction components, acting respectively on the cohesive fraction and on the cracked one; $\chi(\xi)$ is the internal static variable, which governs softening/hardening phenomenon in the damage activation function. The rate of elastic deformations can be obtained from Eqs.(2), (3) and (5) and written in the following form: $\dot{\delta}_{i}^{s e}=\dot{u}_{i}$ and $\dot{\delta}_{i}^{f e}=\dot{u}-\dot{\delta}_{i}^{f p}-\dot{\delta}_{i}^{f d}$. By substitution of Eqs. (10)-(14) and of rate of elastic deformations in Eq.(9), dissipation becomes

$$
D=\sum_{i=1}^{3}\left[\left(t_{i}-t_{i}^{c}-t_{i}^{f}\right) \dot{u}_{i}+t_{i}^{f} \dot{\delta}_{i}^{f p}+Y_{i} \dot{\omega}_{i}\right]+\chi \dot{\xi},
$$

whereas the opening displacement jump $\boldsymbol{\delta}^{f d}$ is active only under null traction conditions and it does not produces any dissipation.

An elastic loading step is characterized by null increment of plastic deformation and damage, that is $\dot{\omega}_{i}=0, \dot{\delta}_{i}^{f p}=0$ for $i=1,2,3 \dot{\xi}=0$; so the dissipation $D$ vanishes and the rate elastic deformations of the two fractions are $\dot{\delta}_{i}^{c e}=\dot{\delta}_{i}^{c e}=\dot{u}_{i}$ and dissipation can be written as follows

$$
D=\sum_{i=1}^{3}\left(t_{i}-t_{i}^{c}-t_{i}^{f}\right) \cdot \dot{u}_{i}=0 .
$$

Since Eq.(16) has to be verified for any elastic loading step $\dot{\boldsymbol{u}}$, the classic internal balance condition is obtained:

$$
t_{i}=t_{i}^{c}+t_{i}^{f}
$$

which states interface traction given as the sum of frictional and cohesive contributions; internal equilibrium equation (17) is strongly satisfied in interface element and it is postulated to hold for any material state, and not only under elastic behaviour. Two-dimensional finite elements which pointwise satisfy equilibrium equations are proposed in [26]. Moreover, the overall interface elastic traction-displacement relation can be obtained by substituting of Eqs.(12) and (13) in Eq.(17) and it is given as

$$
t_{i}=\left(1-\omega_{i}\right) K_{i}^{c} \delta_{i}^{c e}+\omega_{i} K_{i}^{f} \delta_{i}^{f e}
$$




\subsection{Damage activation and evolution laws}

The constitutive model is defined in the framework of non-associative damage theory, with damage evolution governed by the following yield function $\phi_{d}$ and damage potential function $\Omega_{d}$

$$
\begin{aligned}
\phi_{d}\left(Y_{i}, \chi\right) & :=a_{1} Y_{1}+a_{2} Y_{2}+a_{3} Y_{3}-Y_{0}-\chi(\xi) \leq 0 \\
\Omega_{d}\left(Y_{i}, \chi\right) & :=Y_{1}+Y_{2}+Y_{3}-\chi(\xi), \\
\text { with } 0 & <a_{i} \leq 1 .
\end{aligned}
$$

where $a_{i}$ with $i=1,2,3$ are constitutive parameters; under the hypothesis of isotropic behaviour on the tangential plane $\left(\boldsymbol{e}_{1}, \boldsymbol{e}_{2}\right)$ the two corresponding parameter are assumed to be equal $a_{1}=a_{2} . Y_{0} / a_{1}=Y_{0} / a_{2}>0$ and $Y_{0} / a_{3}>0$ are initial yielding threshold respectively in pure mode II debonding condition and in pure mode I condition. The damage variables evolves only when the damage activation function is verified as equality, that is $\phi_{d}=0$, and the increments of damage variables and internal variable are governed by the following flow rules and loading unloading conditions

$$
\begin{aligned}
\dot{\omega}_{i} & =\frac{\partial \Omega_{d}}{\partial Y_{i}} \dot{\lambda}_{d}=\dot{\lambda}_{d} \\
\dot{\xi} & =-\frac{\partial \Omega_{d}}{\partial \chi} \dot{\lambda}_{d}=\dot{\lambda}_{d} \\
\dot{\lambda}_{d} & \geq 0, \quad \phi_{d} \dot{\lambda}_{d}=0, \quad \dot{\phi}_{d} \dot{\lambda}_{d}=0
\end{aligned}
$$

with $\dot{\lambda}_{d}$ non-negative scalar multiplier. Although the damage model is based on three independent damage variables $\omega_{i}$ with $i=1,2,3$, the flow rules in Eqs.(24a, b) produce equal damage increments and the proposed model is equivalent to an isotropic model (i.e. $\omega_{1}=\omega_{2}=\omega_{3}=\omega$ ). Nevertheless, the formal use of three independent damage variables allows us to define the three relevant conjugated thermodynamic forces, the energy release rates defined in Eq.(11), which govern the activation of damage in Eq.(19). The values of the constitutive parameters $a_{1}=a_{2}$ and $a_{3}$ allow us to control the damaging condition and the relevant fracture energy independently for each delamination mode.

\subsection{Frictional plastic activation and evolution laws}

Activation and evolution of frictional plastic deformation components $\boldsymbol{\delta}^{f p}$ is developed by the same approach proposed in [29, 31], which is defined in the framework of non-associative plasticity theory, and it is governed by the classical Mohr-Coulomb yield function

$$
\phi_{p}:=\sqrt{t_{1}^{f^{2}}+t_{2}^{f^{2}}}+c_{f} t_{3}^{f} \leq 0 .
$$

and by means of the following plastic potential

$$
\Omega_{p}:=\sqrt{t_{1}^{f^{2}}+t_{2}^{f^{2}}}+c_{d} t_{3}^{f},
$$


where $c_{f} \geq c_{d}$ are the frictional and the dilatancy coefficients respectively. The plastic flow rules and loading/unloading conditions are

$$
\begin{aligned}
& \dot{\delta}_{1}^{f p}=\frac{\partial \Omega_{p}}{\partial t_{1}^{f}} \dot{\lambda}_{p}=\frac{t_{1}^{f}}{\sqrt{t_{1}^{f^{2}}+t_{2}^{f^{2}}}} \dot{\lambda}_{p}, \\
& \dot{\delta}_{2}^{f p}=\frac{\partial \Omega_{p}}{\partial t_{2}^{f}} \dot{\lambda}_{p}=\frac{t_{2}^{f}}{\sqrt{t_{1}^{f^{2}}+t_{2}^{f^{2}}}} \dot{\lambda}_{p}, \\
& \dot{\delta}_{3}^{f p}=\frac{\partial \Omega_{p}}{\partial t_{3}^{f}} \dot{\lambda}_{p}=c_{d} \dot{\lambda}_{p}, \\
& \dot{\lambda}_{p} \geq 0, \quad \phi_{p} \dot{\lambda}_{p}=0, \quad \dot{\phi}_{p} \dot{\lambda}_{p}=0 .
\end{aligned}
$$

Finally, in order to prevent tensile frictional traction $\left(t_{3}^{f}>0\right)$, once the plastic correction produces a positive normal elastic frictional deformation $\left(\delta_{3}^{f e}>0\right)$, the detachment deformation is assigned as $\boldsymbol{\delta}^{f d}=\mathbf{u}_{f}-\boldsymbol{\delta}^{f p}$, so that the frictional elastic deformation in Eq.(3) is identically null $\left(\boldsymbol{\delta}^{f e}=\mathbf{0}\right)$ and the kinematic conditions in Eq.(4) are fulfilled.

\subsection{Thermodynamic consistency}

The second principle of thermodynamics requires positive dissipation for any damaging or plastic loading step. When only damage activation function is attained, that is $\phi_{d}=0$ and $\phi_{p}<0$, damage evolves without any plastic increment $\left(\dot{\omega}_{i}=\dot{\xi}=\dot{\lambda}_{d}>0, \dot{\delta}_{i}^{f p}=0, i=1,2,3\right)$ and, by substitution in Eq.(15) and assuming that the internal equilibrium condition in Eq.(17) holds, the relevant damage dissipation rate is

$$
D_{d}=\sum_{i=1}^{3} Y_{i} \dot{\omega}_{i}-\chi \dot{\xi} \geq \sum_{i=1}^{3} a_{i} Y_{i} \dot{\lambda}_{d}-\chi \dot{\lambda}_{d}=Y_{0} \dot{\lambda}_{d} \geq 0 .
$$

where the Equations (19)-(24) have been considered. Equation (31) shows positiveness of dissipation rate for any increment of damage.

For loading steps with only plastic activation $\left(\phi_{d}<0\right.$ and $\left.\phi_{p}=0\right)$ damage increment is null and plastic increments are given by Eqs.(30). The relevant rate dissipation is given by substitution of Eqs.(25), (26) and (30) in Eq.(9), that is

$$
D_{p}=\mathbf{t}^{f} \cdot \dot{\boldsymbol{\delta}}^{f p}=\left(\sqrt{{t_{1}^{f^{2}}+t_{2}^{f^{2}}}^{2}}+c_{d} t_{3}^{f}\right) \dot{\lambda}_{p}>\left(\sqrt{{t_{1}^{f^{2}}+t_{2}^{f^{2}}}^{2}}+c_{f} t_{3}^{f}\right) \dot{\lambda}_{p}=0,
$$

which shows positive dissipation for any plastic increment.

The total rate dissipation, for a general loading step, is the sum of the plastic contribution and of the damaging one

$$
D=D_{p}+D_{d} \geq 0
$$


which is null for any elastic loading step and is positive for any increment of damage or for any increment of the plastic components, satisfying the second thermodynamic law.

The cohesive model is completed by the softening law, which governs the evolution of the static internal variable, and the initial yielding threshold $Y_{0}$ in the damage activation function in Eq.(19). In the case of linear softening behaviour the static internal variable is

$$
\chi(\xi):=Y_{0}\left[((1-\xi)+c \xi)^{-2}-1\right]
$$

where $c<1$ is the ratio between limit elastic separation displacement and fully debonding separation displacement. That is $c=u_{e} / u_{f}$ where: $u_{e}$ is the limit elastic separation displacement and $u_{f}$ is the separation displacement at the fully debonding condition. Different softening laws can be considered; for instance a logarithmic cohesive law has been proposed in [5].

\subsection{Constitutive parameters}

The proposed non-associative damage model is based on a set of constitutive parameters some of which are not independent. The yield function in Eq.(19) is defined by the constitutive parameters $a_{1}=a_{2}=a_{t}, a_{3}=a_{n}$ and $Y_{0}$ but only two of them are independent each other.

In accordance with experimental data, only the case of mode II fracture energy greater then the mode I value $\left(G_{I I}>G_{I}\right)$ is analysed by assuming the following constitutive parameters of damage activation function in Eq.(19): $a_{t}<1, a_{n}=1$. Moreover, the constitutive parameter $Y_{0}$ is defined as well as proposed in [29], namely $Y_{0}=1 / 2 K_{3}^{s} u_{e}^{2}$.

The proposed interface cohesive zone model produces bilinear response in the traction separation displacement diagram for pure mode I and pure mode II debonding condition, as shown in Fig. 4. In the pure mode I debonding condition the limit elastic displacement is $\bar{u}_{I}^{e}=u_{e}$, the fully debonding displacement is $\bar{u}_{I}^{f}=u_{f}$ and the maximum normal traction is $\bar{t}_{I}=K_{3}^{c} u^{e}$. In the pure mode II debonding condition, under null normal traction component $t_{3}=0$, limit elastic displacement $\bar{u}_{I I}^{e}$, fully debonding displacement $\bar{u}_{I I}^{f}$ and maximum tangential traction $\bar{t}_{I I}$, respectively are

$$
\begin{aligned}
\bar{u}_{I I}^{e} & =b u_{e} \\
\bar{u}_{I I}^{f} & =b u_{f} \\
\bar{t}_{I I} & =b K_{1}^{s} u_{e}
\end{aligned}
$$

where $b=\sqrt{K_{3}^{s} / a_{t} K_{1}^{s}}$.

In Fig. 4 the qualitative response of the interface subjected to the monotonic loading path is depicted in terms of traction vs separation displacement for the two limit cases of pure mode I and pure mode II and for a mixed mode delamination condition. In Fig. 4 it is also plotted the qualitative response of the interface subjected to the monotonic tangential loading path, under constant 


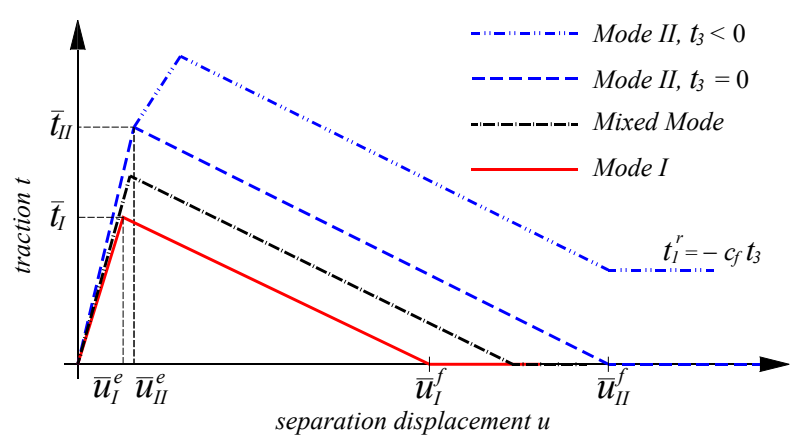

Figure 4: Qualitative response of the CZM for mode I, mode II and mixed mode delamination condition, under monotonic loading path, in terms of traction and separation displacement.

compressive normal traction $\left(t_{3}<0\right)$, showing the effect of frictional behaviour in the decohesion process and the residual tangential frictional traction $t_{1}^{r}=$ $-c_{f} t_{3}$. Finally, the mode I fracture energy is

$$
G_{I}=\frac{1}{2} K_{3}^{s} \bar{u}_{I}^{e} \bar{u}_{I}^{f}=\frac{1}{2} K_{3}^{s} u_{e} u_{f}
$$

and the mode II fracture energy is

$$
G_{I I}=\frac{1}{2} k_{1}^{s} \bar{u}_{I I}^{e} \bar{u}_{I I}^{f}=G_{I} / a_{t}>G_{I}
$$

The mixed mode fracture energy $G_{m x}$ is $G_{I} \leq G_{m x} \leq G_{I I}$ and the constitutive parameter $a_{t}$ is the ratio between mode I and mode II fracture energies $a_{t}=$ $G_{I} / G_{I I}<1$, for the assumed value $a_{n}=0$.

Several experimental investigations confirm that fracture energy in mixed mode debonding condition gradually increases from the pure mode I value $G_{I}$ to the pure mode II value $G_{I I}$. Such a result is reported by Benzeggagh and Kenane in [4], who measured the fracture energy of a unidirectional glass/epoxy composite for six different mixed mode conditions, by the mixed mode bending apparatus developed by Crews and Reeder in [9]. In [4] the pure mode I fracture energy has also been measured by a set of double cantilever beam (DCB) tests and the pure mode II fracture energy has been measured by a set of End Loaded Split (ELS) tests.

\section{Numerical simulations}

\subsection{Benzeggagh and Kenane experimental tests}

The proposed interface CZM has been implemented in the finite element code FEAP [38] and, in order to validate its effectiveness, the delamination experimental tests developed by Benzeggagh and Kenane [4] have been numerically simulated with a unique set of constitutive parameters. The material used for the specimen tested in [4] was a E-glass fibres reinforced composite 
with epoxy resin, and it can be modelled as an orthotropic elastic material. All specimens were monitored with strain gauge and acoustic emission transducer for the damage initiation detection. The acoustic emission transducer have also been used in [19] for ultrasonic inspection and detection of debonding defects between CFRP-reinforcement and concrete. In order to obtain the same elastic stiffness between numerical simulations and experimental data, the longitudinal elastic moduli and the tangential ones assumed for the numerical simulations have been reduced of $25 \%$, with respect to the experimental values reported in [4]. These different values could be due the difference between the tensile and flexural elastic moduli of composite materials. In fact elastic moduli are usually evaluated in uniaxial tensile tests whereas the delamination tests involve the flexural behaviour.

The total fracture resistance obtained from the experimental test in [4] in pure mode I delamination condition is $G_{I R}=0.429 \pm 0.042 \mathrm{~N} / \mathrm{mm}$ and in pure mode II delamination condition is $G_{I I R}=2.905 \pm 0.244 \mathrm{~N} / \mathrm{mm}$.

In [4] several tests have been developed for each mixed mode ratio $G_{I I} / G_{T}$, with some different values of pre-existing crack $a_{0}$, but the results of the experimental tests are plotted in terms of force vs displacement only for one value of initial crack $a_{0}$.

The numerical simulations proposed in the present paper have been performed with the same values of initial crack $a_{0}$ of the specimens whose results are plotted in [4] in terms of force and displacement. The specimen domain has been discretized by use of nine-node two-dimensional finite elements with orthotropic linear elastic material, under plane strain conditions. The delamination surface has been discretized by use of six-nodes interface finite elements.

The numerical solutions of the non-linear loading steps are performed by Newton-Rapson iterative method and by use of unsymmetric solver. The loading condition is defined in terms of imposed displacement with increments of $\Delta u=0.1 \mathrm{~mm}$ and the convergence tolerance is fixed as tol $=10^{-10}$ in terms of deformation energy ratio between the current iteration step and the first iteration step.

Geometry and sizes of the mode I, mode II and mixed mode delamination tests are, respectively represented in the Figures $5 \mathrm{a}, \mathrm{b}, \mathrm{c}$. The mixed mode ratio is defined as a function of the distance $d$ in the mixed mode bending apparatus developed by Crews and Reeder in [9] and represented in Fig.5c. The values of distance $d$ and relevant values of the mixed mode ratio considered for the numerical simulations are collected in the following Table 1 . The numerical simulations of the MMB tests have been performed by discretization of the end notched specimen and of the loading beam. The loading beam has been constitutively modelled with a much stiffer linear elastic material than the specimen. The two loading points between specimen and loading beam have been mutually connected through master slave multi-freedom constrains.

The set of constitutive parameters of the proposed interface constitutive model adopted for all the numerical simulations are collected in Table 2.

In Fig.6 numerical and experimental results of the DCB test are compared to the analytical solution based on the Beam and Fracture Mechanics (BFM) 
theories (see [12]) in terms of opening displacement $u$ vs applied load $P$. The delamination curves of the DCB test, plotted in Fig.6 and obtained with the mode I experimental value of fracture energy $G_{I}=0.429 \mathrm{~N} / \mathrm{mm}$, show a very good agreement between the analytical solution and the numerical one, expect that in the initial elastic branch; motivations of the differences between analytical and numerical solutions have already been analysed in [31]. Although the analytical and numerical solutions obtained with the experimental value $G_{I}=0.429 \mathrm{~N} / \mathrm{mm}$ are in mutual agreement, they cannot reproduce the relevant experimental curve with good accuracy, showing a lower strength. The mode I experimental value of fracture energy $G_{I}=0.429 \mathrm{~N} / \mathrm{mm}$ would seem underestimated for the experimental delamination curve obtained in [4]. Therefore, in order to obtain the best fitting between numerical and experimental results (see Fig.6), the mode I fracture energy considered in the numerical simulations is $G_{I}=0.833 \mathrm{~N} / \mathrm{mm}$, which is greater than the total fracture resistance $G_{I R}=0.429 \pm 0.042 \mathrm{~N} / \mathrm{mm}$, experimentally evaluated in [4].

\begin{tabular}{|l|l|l|l|l|l|}
\hline \hline Distance $d$ & $90 \mathrm{~mm}$ & $60 \mathrm{~mm}$ & $50 \mathrm{~mm}$ & $40 \mathrm{~mm}$ & $30 \mathrm{~mm}$ \\
mixed mode ratio $G_{I I} / G_{T}$ & $28 \%$ & $43 \%$ & $53 \%$ & $72 \%$ & $91 \%$ \\
\hline \hline
\end{tabular}

Table 1: Values of distance $d$ and corresponding mixed mode ratio of MMB experimental tests and numerical simulations

\begin{tabular}{|l|l|}
\hline \hline & Cohesive Parameters \\
\hline Normal elastic stiffness & $K_{n}^{c}=1666.6 \mathrm{~N} / \mathrm{mm}^{3}$ \\
Tangential elastic stiffness & $K_{t}^{c}=500000 \mathrm{~N} / \mathrm{mm}^{3}$ \\
Constitutive parameter & $a_{n}=1$ \\
Constitutive parameter & $a_{t}=0.286786$ \\
Mode I elastic displ. & $\bar{u}_{I}^{e}=u_{e}=0.001 \mathrm{~mm}$ \\
Mode I debonding displ. & $\bar{u}_{I}^{f}=u_{f}=1 \mathrm{~mm}$ \\
Tensile strength & $\bar{t}_{I}=1.666 \mathrm{~N} / \mathrm{mm}^{2}$ \\
Mode II elastic displ. & $\bar{u}_{I I}^{e}=1.091 \cdot 10^{-4} \mathrm{~mm}$ \\
Mode II debonding displ. & $\bar{u}_{I I}^{f}=0.1091 \mathrm{~mm}$ \\
Shear strength & $\bar{t}_{I I}=54.5 \mathrm{~N} / \mathrm{mm}$ \\
Mode I Fracture energy & $G_{I}=0.8333 \mathrm{~N} / \mathrm{mm}$ \\
Mode II Fracture energy & $G_{I I}=2.90576 \mathrm{~N} / \mathrm{mm}^{\prime}$ \\
\hline & Frictional Parameters \\
\hline Normal elastic stiffness & $K_{n}^{f}=1666.6 \mathrm{~N} / \mathrm{mm}^{3}$ \\
Tangential elastic stiffness & $K_{t}^{f}=25000 \mathrm{~N} / \mathrm{mm}^{3}$ \\
Frictional coefficient & $c_{f}=\tan 35^{\circ} \approx 0.7$ \\
Dilatancy coefficient & $c_{d}=0$ \\
\hline
\end{tabular}

Table 2: Constitutive parameters used for the numerical simulations. 
a)

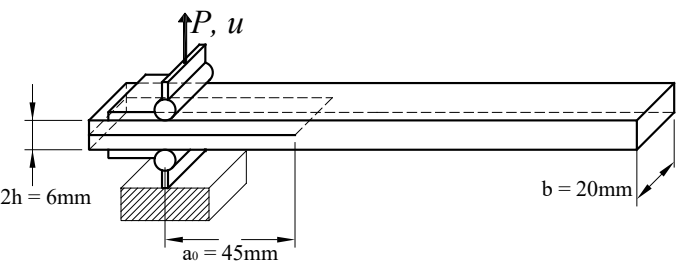

b)

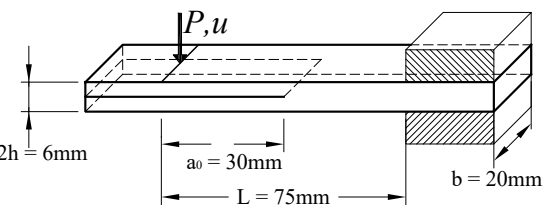

c)

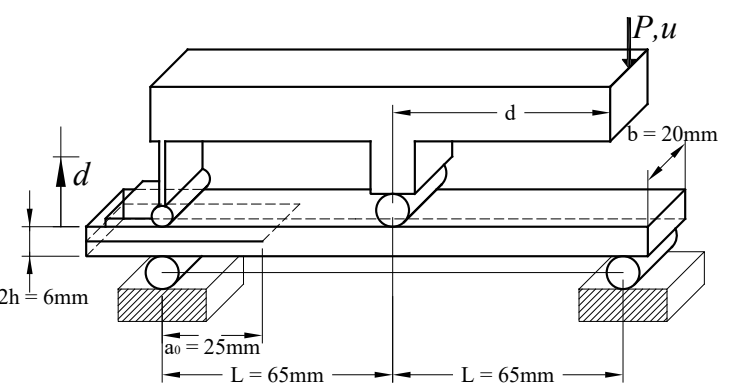

Figure 5: Sizes and geometry of specimen for the three different delamination conditions: a) DCB test for pure mode I delamination; b) ELS test for pure mode II delamination; c) MMB test for the mixed mode delamination.

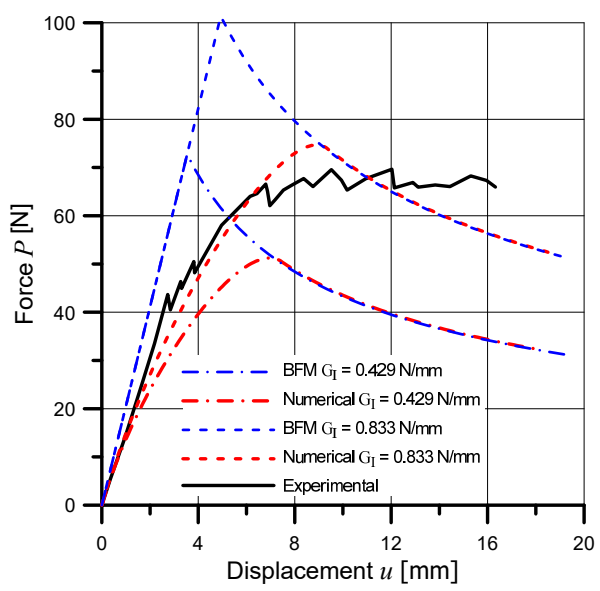

Figure 6: Results of the DCB test in terms of applied load $P$ vs opening displacement $u$ of: experimental test, numerical simulations with two different fracture energies and the relevant analytical BFM solutions. 
The results of the numerical solutions of the pure mode I DCB test and the results of the mixed mode bending numerical simulations, all of which performed with the interface constitutive parameters collected in Table 2, are compared to the relevant experimental results in terms of applied force $P$ vs displacement $u$ in the Fig.7.

Finally, the results of the pure mode II delamination test ELS, represented in Fig. 5c, are plotted in Fig.8a, in terms of applied force $P$ and displacement $u$, and compared to the results of the ELS experimental test developed by Benzeggagh and Kenane in [4].

The proposed cohesive-frictional constitutive formulation is able to model also the transition from the initial cohesive behaviour, of the virgin material, to the residual frictional one of the fully debonded interface. Therefore the presence of frictional effects between the delamination surfaces can be effectively analysed. The frictional phenomenon can develop only in the ELS test, due to the compressive state of stress between the two delaminated surfaces, and the results of the delamination test with a frictional coefficient $c_{f}=\tan \left(35^{\circ}\right) \approx 0.7$ are compared to the frictionless results and to the experimental results in Fig. 8, showing a small effect for the ELS test in terms of peak load level. In order to clarify the frictional behaviour modelled by the proposed formulation, two unloading-reloading cycles have been numerically simulated after the post-peak drop related to the full delamination. In the frictionless response $\left(c_{f}=0\right)$ the unloading curves coincide to the reloading ones, without any frictional energy dissipation. On the contrary, the frictional behaviour can be observed in the numerical solution with frictional coefficient $\left(c_{f} \approx 0.7\right)$, where the unloadingreloading cycles produce two frictional dissipation cycles with unloading paths different than the reloading ones. The detail of the two unloading-reloading cycles is plotted in Fig. $8 \mathrm{~b}$.

The two Figs 7 and 8 a show that the proposed non associative damage model (NADM) can reproduce with good accuracy, with of a unique set of constitutive parameters, several delamination tests with a full range of modal ratio $0 \leq G_{I I} / G_{T o t} \leq 1$. Figure 9 plots the total fracture energies obtained by the proposed model for the full range of modal ratio $0 \leq G_{I I} / G_{T o t} \leq 1$, compared to the relevant experimental values obtained in [4]. The averages of experimental data, for each value of the modal ratio, are connected by the dotted line with an almost linear variation. On the contrary, the proposed NADM produces a non-linear variation of the total fracture energy with the modal ratio.

The maps of normal stress $\sigma_{x x}$ obtained by the DCB test, by the five MMB tests and by the ELS test (with frictionless interface) are plotted in the Figures $10 \mathrm{a}, \mathrm{g}$. The maps of tangential stress $\sigma_{x y}$ obtained by the DCB test, for the five MMB tests and for the ELS test (with frictionless interface) are plotted in the Figs. $11 \mathrm{a}$, g. The maps of normal and tangential stresses have been plotted at the loading step corresponding to the imposed displacement $u=10 \mathrm{~mm}$ for all the numerical analyses. Figures $10 \mathrm{~b}$, f and the Figs. $11 \mathrm{~b}, \mathrm{f}$ show also the finite element discretization of the loading beam of the MMB apparatus and the position of loading points between specimen and loading bar. 


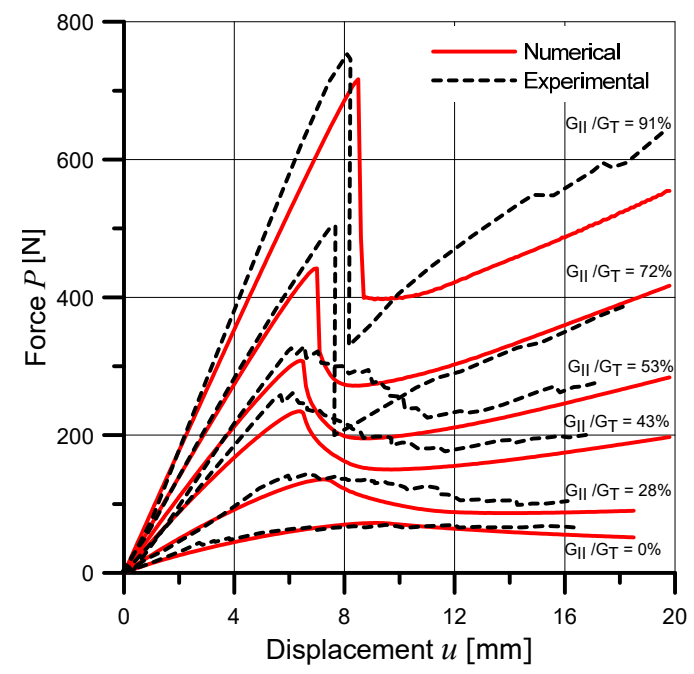

Figure 7: Results of experimental tests and numerical simulations of the DCB test and mixed mode delamination tests in terms of applied load $P$ vs opening displacement $u$.

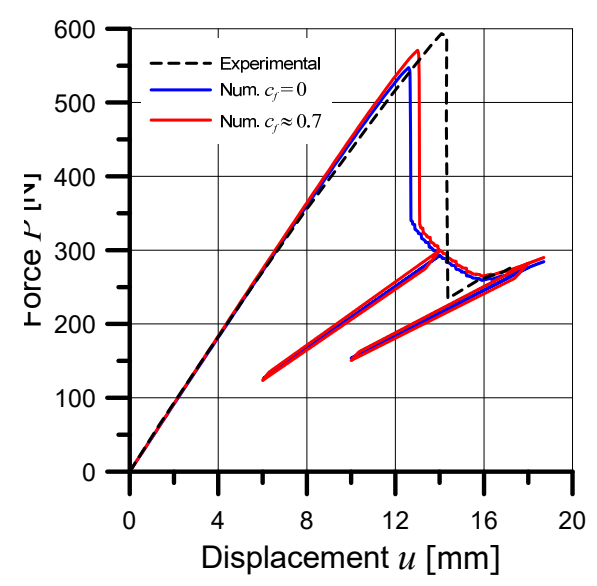

a)

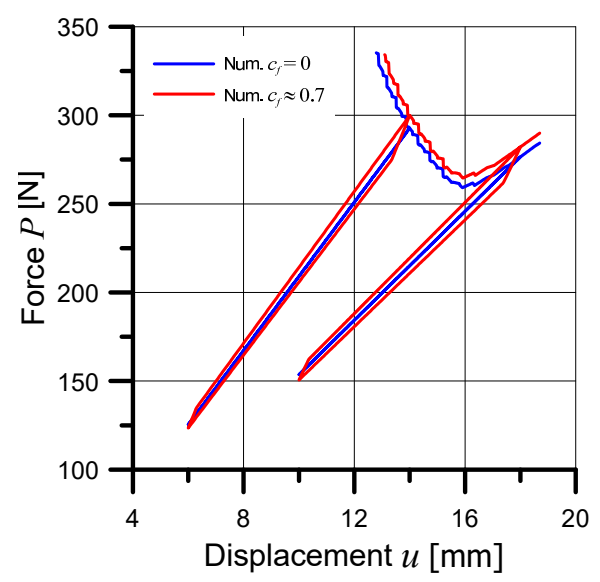

b)

Figure 8: Results of experimental test and numerical simulations of the ELS test, in terms of applied load $P$ and opening displacement $u$. Results of numerical simulations are with frictionless interface $\left(c_{f}=0\right)$ and with frictional interface $\left(c_{f}=\tan \left(35^{\circ}\right) \approx 0.7\right)$ : a) complete numerical responses; b) detail of the two unloading-reloading cycles of the two numerical simulations, showing the effect of the friction. 


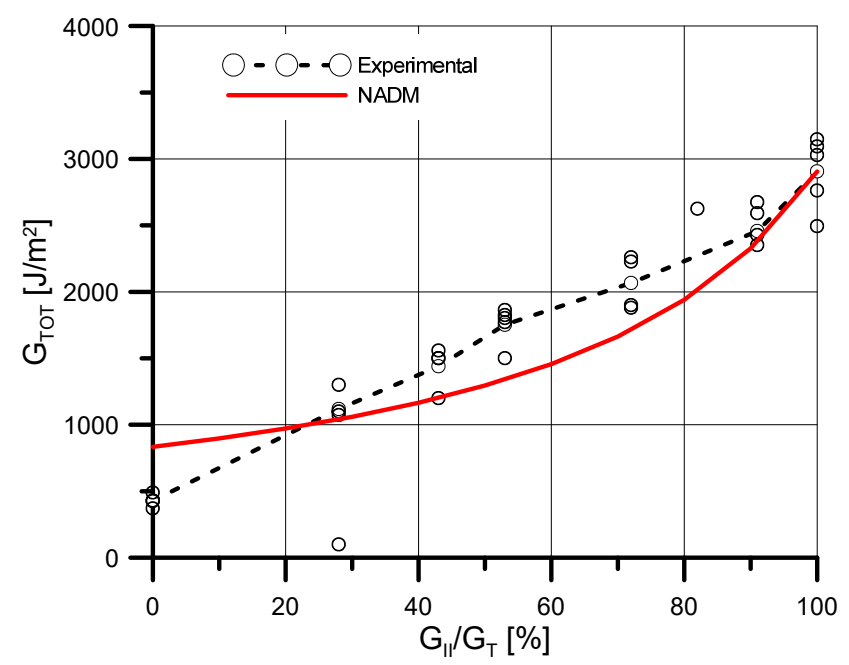

Figure 9: Total fracture energies $G_{T \text { ot }}$ obtained by the NADM for the full range of modal ratio $0 \leq G_{I I} / G_{T} \leq 1$, compared to the relevant experimental values. The averages of experimental values, for each value of the modal ratio, are connected by the dotted line.

The three-dimensional numerical simulation of the DCB test for pure mode I delamination condition has been also performed by use of twenty-seven node Lagrangian brick elements with quadratic shape functions and elastic orthotropic constitutive model for the bulk. The delamination surface has been modelled with eighteen node interface elements, with quadratic interpolation functions and with the constitutive model proposed in the present paper. The domain is discretized by 1500 brick elements, 225 interface elements and 15708 nodes. The results of the three-dimensional numerical simulation are represented in the Fig.12 in terms of opening displacement vs applied force and the results are compared to the responses obtained by the two-dimensional analyses under plane stress and plane strain conditions, with the relevant analytical solutions based on the beam theory and elastic-fracture mechanics theory (BFM). The maps of normal stress $\sigma_{x x}$ obtained by the three-dimensional numerical simulation, for the imposed displacements $u=10 \mathrm{~mm}$ and $u=20 \mathrm{~mm}$, are plotted respectively in the Figs.13 a, b. The evolution of the damage variable $\omega=\omega_{i}$ on the surface $\Gamma$ of the interface is represented in Fig.14 in the undeformed configuration of the lower lamina at several loading steps, corresponding to the following imposed displacement: $u=2,4,6,8,10,12,14 \mathrm{~mm}$. The maps of damage show a not uniform delamination front along the sample width ( $x_{3}$ direction), nevertheless the three-dimensional response does not differ significantly from the two-dimensional responses, as shown in Fig. 12 in terms of applied force and opening displacement. The tree-dimensional analysis is therefore not fully necessary for the analysis of the DBC test, but it is useful to confirm the robustness and the correct implementation of the proposed model. 
a)

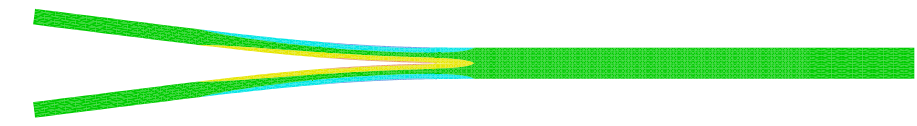

b)

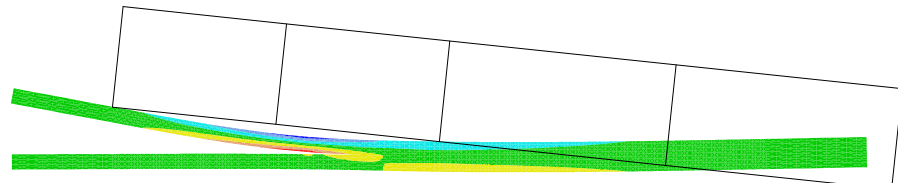

c)

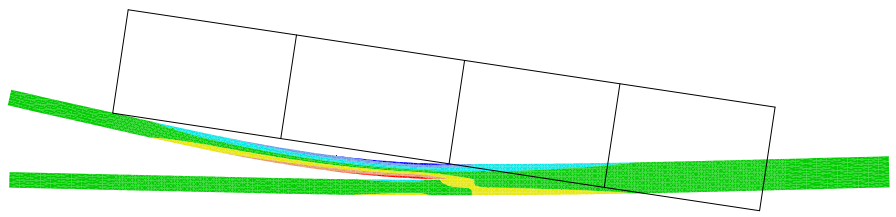

d)

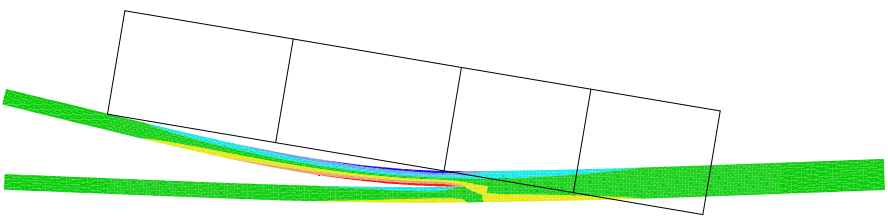

$-250.00$

e)

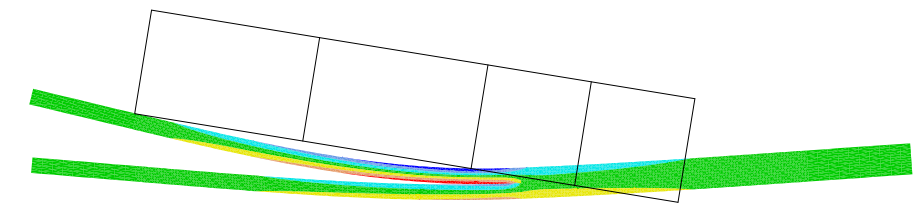

f)

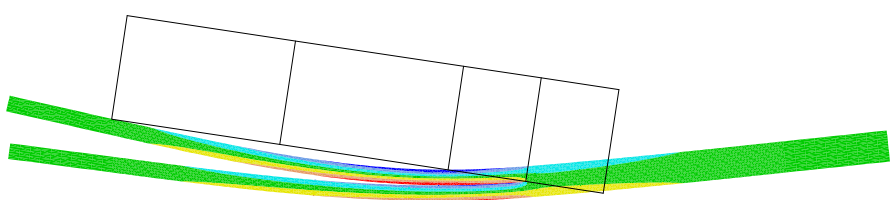

g)

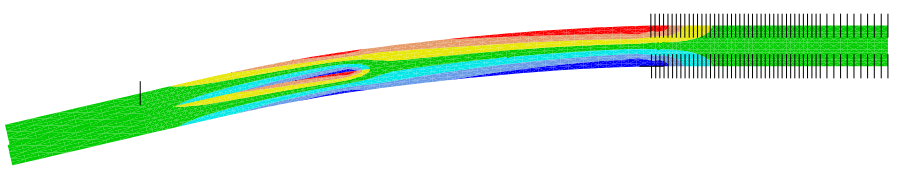

Figure 10: Maps of normal stress $\sigma_{x x}$ obtained for the DCB test, for the five MMB tests and for the ELS test (with frictionless interface), at the loading step corresponding to the imposed displacement $u=10 \mathrm{~mm}$. 
a)

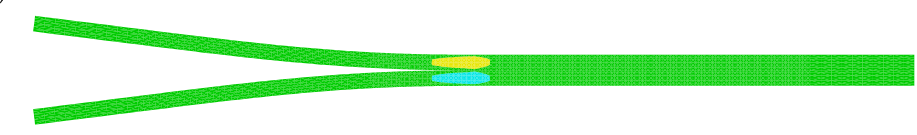

b)

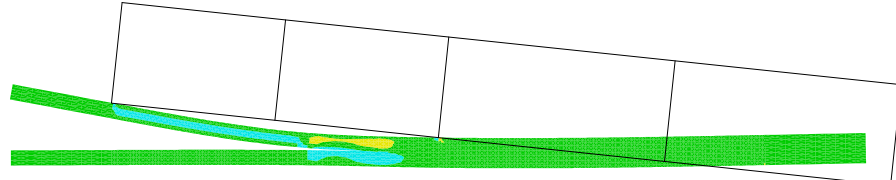

c)

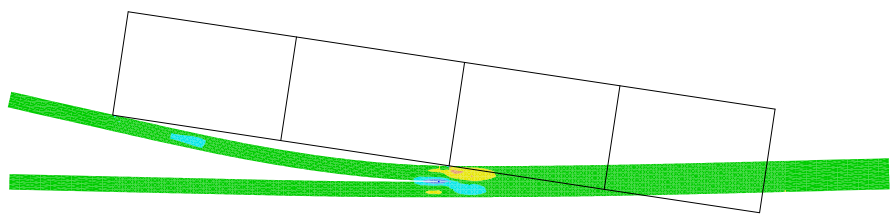

d)

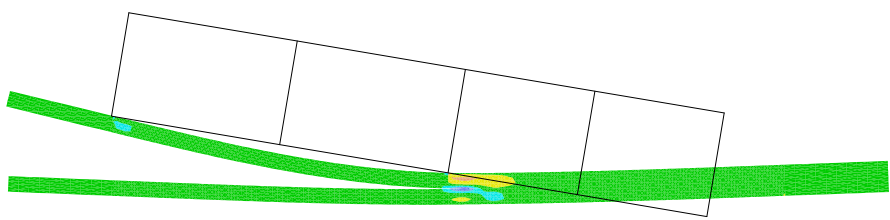

$-25.000$

e)

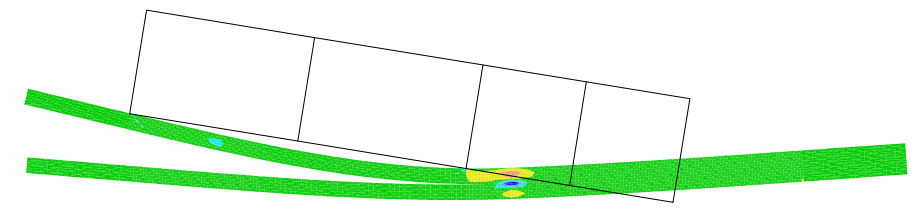

f)

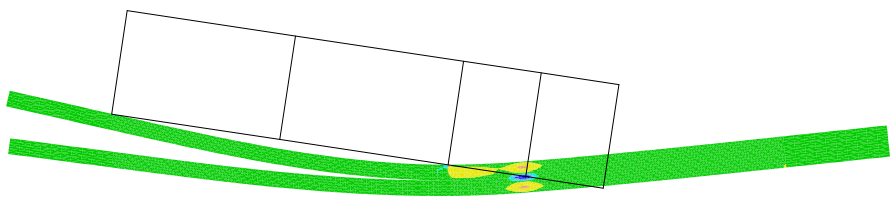

g)

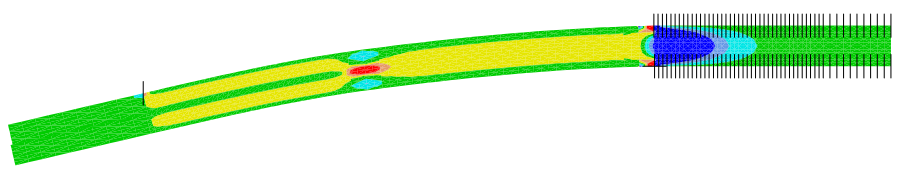

Figure 11: Maps of tangential stress $\sigma_{x y}$ obtained for the DCB test, for the five MMB tests and for the ELS test (with frictionless interface), at the loading step corresponding to the imposed displacement $u=10 \mathrm{~mm}$. 


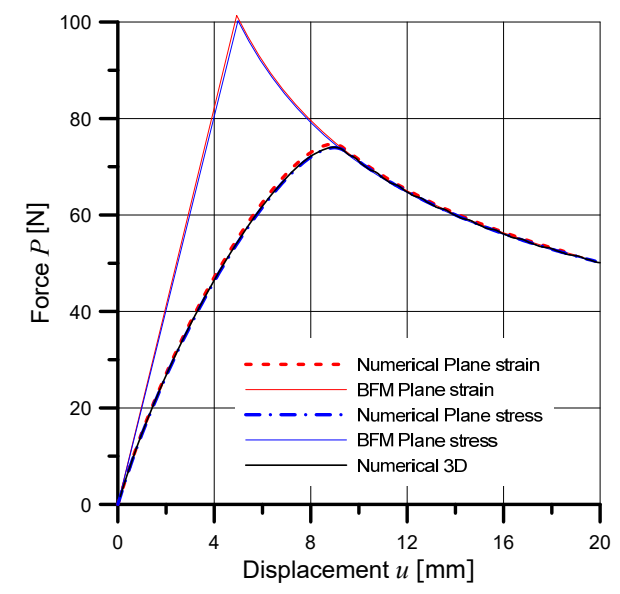

Figure 12: Results of the DCB test in terms of applied load $P$ vs opening displacement $u$ of: experimental test, two-dimensional and three-dimensional numerical simulations and the analytical BFM solutions.

\subsection{Split shear torsion test}

The last numerical simulation regards a quite interesting problem of fracture mechanics, the split shear torsion (SST) test, which has been used in $[18,17]$ for the experimental evaluation of the mode III delamination toughness. The specimens subjected to SST test were obtained from a panel of unidirectional 26 ply IM7 $/ 977-3$ with nominal thickness of $2 \mathrm{~h}=3.3 \mathrm{~mm}$. The specimens were manufactured with an initial delamination area, with the two ends bonded to rectangular steel load tabs, one of which was fixed against rotations and translations and the second one was free only to translate vertically. The schematic representations of the SST test with the tangential splitting displacement $U$ and sizes of specimens tested in $[18,17]$ is shown in Fig.15.

In the papers $[18,17]$ three different values of initial delamination lengths, measured as distance between middle axis of loading tabs and the delamination front, have been tested showing a considerable dependence of the critical toughness from the initial delamination length. In the present numerical simulation, only the first delamination length $a=31.8 \mathrm{~mm}$ is considered. The two loading tabs have not been discretized in the numerical simulation, the specimen has been discretized starting from the middle axis of the loading tabs and the two ends of the two delaminated arms have been constrained. In order to take into account the additional stiffness produced by the loading tabs and to numerically obtain the same stiffness of the experimental data, the initial delamination length has been corrected to the following value $a_{c}=29.3 \mathrm{~mm} \neq a$. The bulk of the specimens has been modelled as elastic and orthotropic with the same constitutive parameter reported in [18]. The specimen has been discretized with 2000 twenty-seven node brick elements, with 400 eighteen node interface elements and with 21730 nodes. 

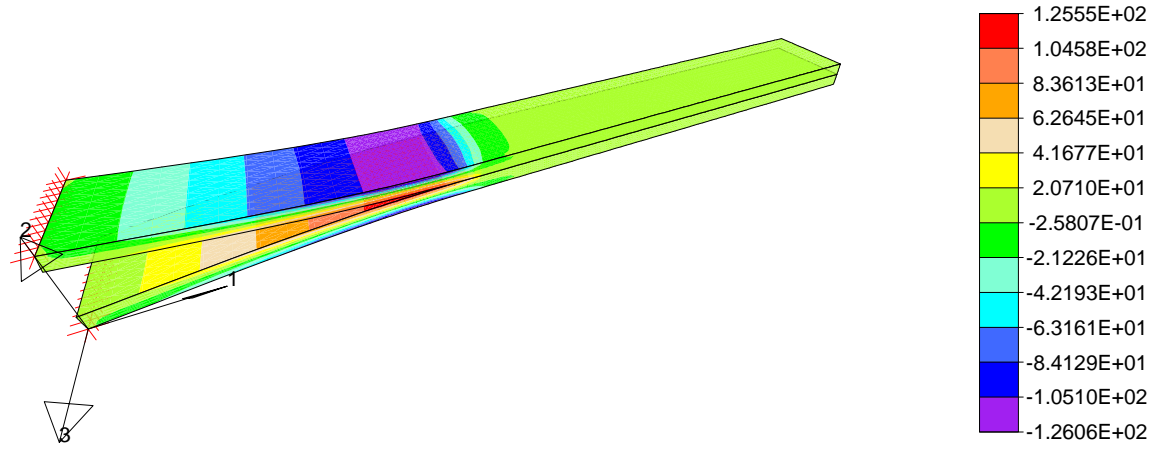

a)

STRESS 1

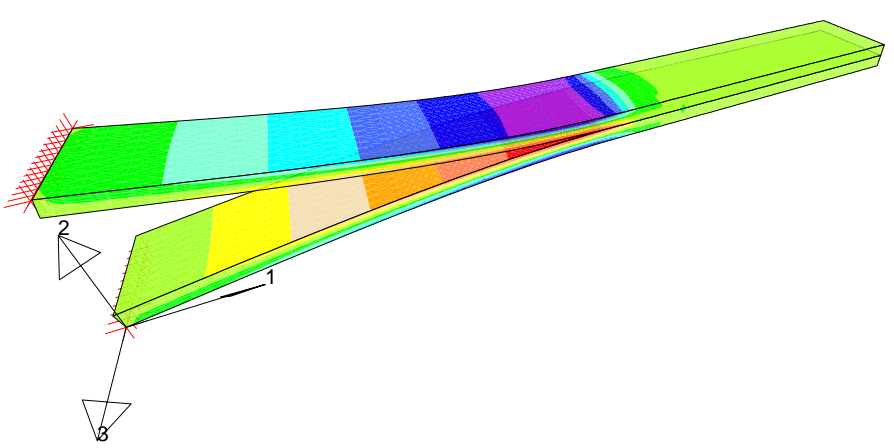

$1.3254 \mathrm{E}+02$ $1.1043 \mathrm{E}+02$ $8.8307 \mathrm{E}+01$ $6.6188 \mathrm{E}+01$ $4.4070 \mathrm{E}+01$ 2. $1951 \mathrm{E}+01$ $-1.6758 \mathrm{E}-01$ $-2.2286 E+01$ $-4.4405 E+01$ $-6.6524 \mathrm{E}+01$ $-8.8642 E+01$ $-1.1076 \mathrm{E}+02$ b)

Figure 13: Maps of normal stress $\sigma_{x x}$ obtained for the three-dimensional DCB test, at the loading step corresponding to the following imposed displacement: a) $u=10 \mathrm{~mm}$, b) $u=$ $20 \mathrm{~mm}$. 


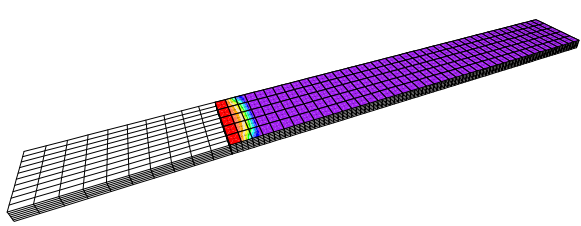

a)

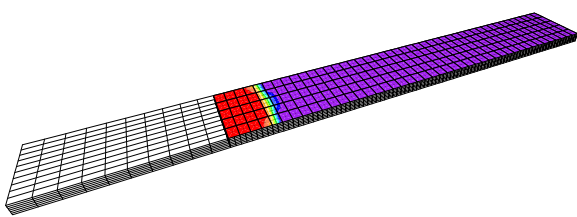

c)

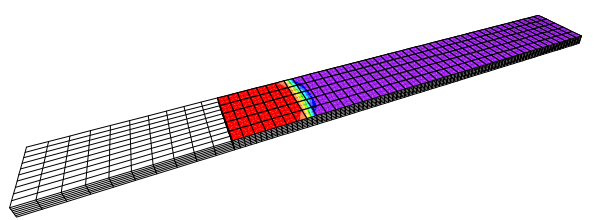

e)

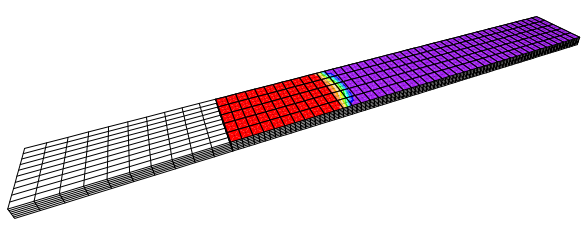

g)

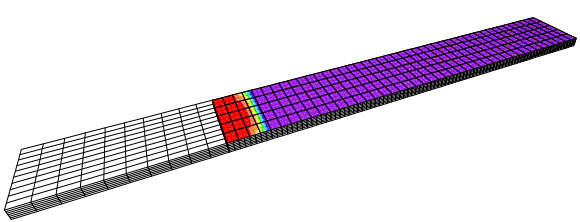

b)

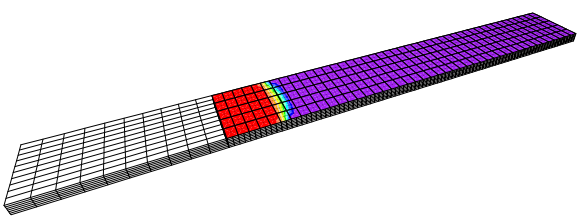

d)

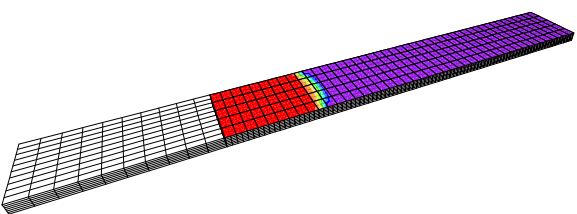

f)

DAMAGE

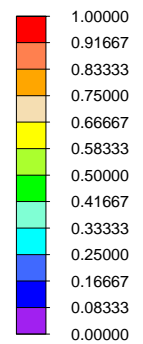

Figure 14: Maps of damage on the surface $\Gamma$ of the interface, obtained for the three dimensional analysis of DCB test, at seven different loading steps corresponding to the following imposed displacements: a) $u=2 \mathrm{~mm}$, b) $u=4 \mathrm{~mm}$, c) $u=6 \mathrm{~mm}$, d) $u=8 \mathrm{~mm}$, e) $u=10 \mathrm{~mm}, \mathrm{f}$ ) $u=12 \mathrm{~mm}, \mathrm{~g}) u=14 \mathrm{~mm}$ 
a)

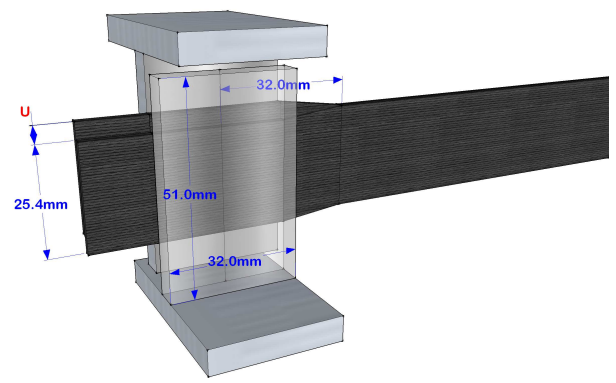

b)

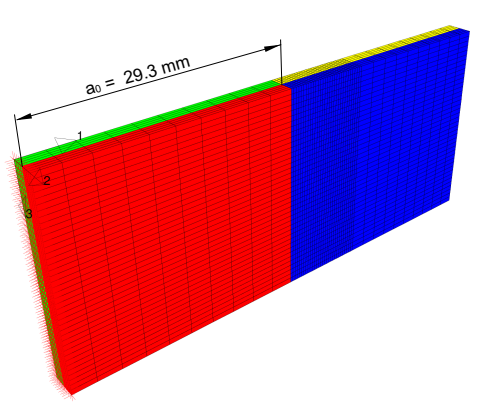

Figure 15: SST test: a) Schematic representation of the SST loading test; b) mesh of the numerical simulation with the boundary conditions.

The apparent mode III delamination toughness obtained in [18] as results of the SST experimental tests ranges from $0.7 \mathrm{~N} / \mathrm{mm}$ to $0.9 \mathrm{~N} / \mathrm{mm}$. The proposed interface constitutive model, with isotropic behaviour on the tangential plane cannot distinguish between mode II and mode III fracture energy, so for this simulation the mode II fracture toughness has been fixed as $G_{I I}=0.8587 \mathrm{~N} / \mathrm{mm}$ and the interface cohesive parameters are: $K_{n}^{c}=K_{t}^{c}=50000 \mathrm{~N} / \mathrm{mm}^{3}, a_{t}=0.25$, $u_{e}=0.00031, u_{f}=0.0277$. The frictional behaviour is not relevant for this problem. The results of the numerical simulation of SST loading test are compared to the experimental data in Fig.16 in terms of loading force $P$ vs imposed displacement $U$, showing a good matching in the loading branch. The numerical unloading path, as result of an elastic-damage behaviour, is a straight branch up to the unloading condition with null load $P=0$ and null displacement $U=0$, whereas the experimental unloading path shows a more complex nonlinear behaviour with residual displacement at the null load condition. This results can be probably caught by considering the interlocking effects between the delaminated surface in a finite displacement framework, but it is not analysed in the present paper. The SST mode III delamination test, as well known in literature, does not produce uniform stress fields at the delamination front and two different delamination modes can be envisaged. In [18, 17] the Energy Release Rate (ERR) for pure mode II and pure mode III delamination conditions have been considered for the SST test, showing that the mode II ERR is negligible except that near the free edges, and the mode III ERR is maximum at the centre of the delamination front. Moreover, the delamination starts at the centre of the specimen. This result is confirmed by proposed non-associative interface model as can be observed in the two maps of interface damage plotted in the Figs. 17a, $\mathrm{b}$, obtained respectively at the loading step $U=0.35 \mathrm{~mm}$ and at the loading step $U=0.65 \mathrm{~mm}$. These results, plotted in the deformed configuration with amplified displacements, show that, at the initial stage, the maximum damage is at the specimen centre and it decreases at the free edges. At the maximum loading condition two peaks of damage emerge at the free edges, produced by the mode II effects, as observed in $[18,17]$. 


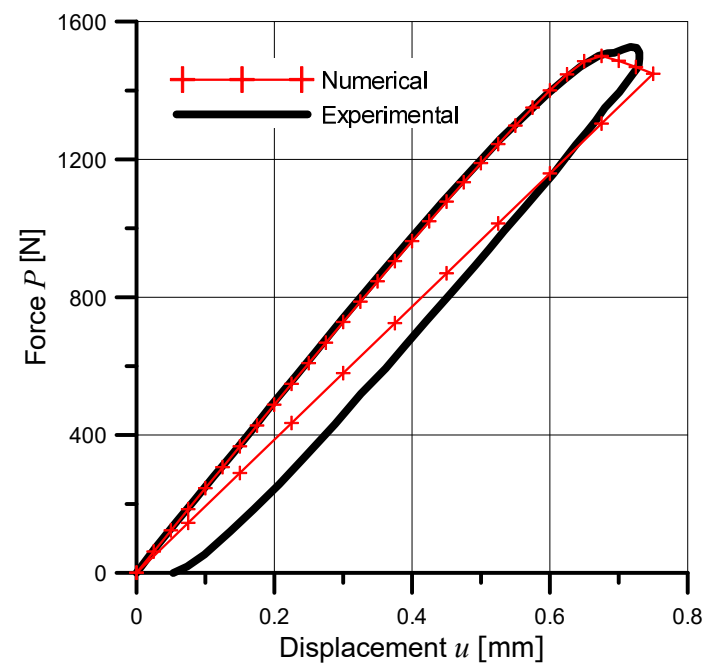

Figure 16: Graphic of numerical simulation results of the SST loading test in terms of in terms of loading force $P$ vs imposed displacement. The results are compared to the experimental data.

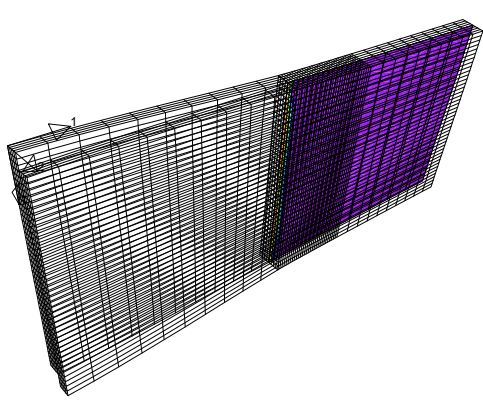

a)

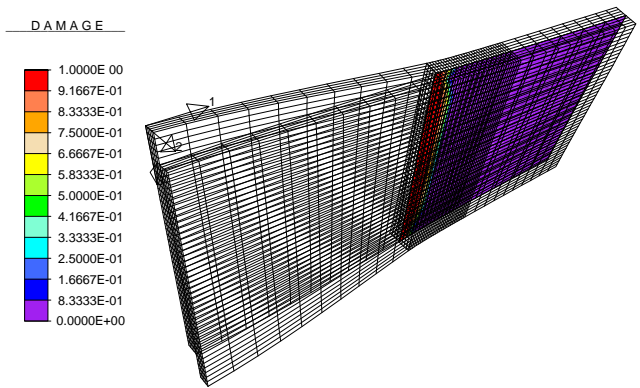

b)

Figure 17: Maps of interface damage for two loading steps of the SST test: a) $U=0.35 \mathrm{~mm}$; b) $U=0.65 \mathrm{~mm}$. 


\section{Conclusions}

The paper proposes an interface unified constitutive framework for modelling cohesive behaviour of the sound interface and for modelling the residual frictional behaviour the debonded interface. The model is based on three independent damage variables and is developed in a consistent thermodynamic framework. The constitutive model is defined through the Helmholtz free energy, the damage activation function and a non-associative dissipation function. The evolution rules are derived in the context of dissipative mechanics with internal variables and produce the same evolution of the three damage variables.

The present paper proposes a new theoretical framework of the interface constitutive models, which allows to model independent damage activation conditions for some different debonding conditions (mode I, mode II or mixed mode). The non-associative flow rules drive the concurrent evolution of the three damage variables and the concurrent degradation of all the traction components in a thermodynamically consistent formulation.

The proposed CZM assumes an isotropic behaviour on the tangential plane and produces two independent fracture energies, $G_{I}$ in pure mode I debonding condition and $G_{I I}$ in pure mode II debonding one. $G_{I}$ and $G_{I I}$ are minimum and maximum values of the work-of-separation for any proportional loading path. The model can also evaluates the presence of frictional tractions both at the fully debonded zones and at the partially debonded ones. The proposed model is able to accurately reproduce, with a unique set of few constitutive parameters, the mechanical response of delamination problems for any mixed mode ratio: pure opening mode, pure sliding mode, mixed mode debonding conditions and closing conditions with the relevant frictional effects.

In order to validate the proposed CZM, model all the experimental tests performed by Benzeggagh and Kenane in [4] have been numerically simulated with a unique set of constitutive parameters. The numerical and experimental results are compared in terms force and displacement, showing good agreement for very different test conditions. Finally, the results of 3D numerical simulation of the SST test for the evaluation of the mode III fracture toughness are proposed showing good agreement both in terms of loading-displacement diagram and in terms of damage evolution on the delamination surface.

\section{Acknowledgements}

The financial support of the Italian Ministry for University and Research (MIUR), under the Grant PRIN-2015, Project No. 2015LYYXA8, "Multiscale mechanical models for the design and optimization of microstructured smart materials and metamaterials" is gratefully acknowledged.

[1] Alfano, G., Sacco, E., 2006. Combining interface damage and friction in a cohesive-zone model. Int. J. Num. Meth. Eng. 68 (5), 542-582. 
[2] Baek, H., Park, K., 2018. Cohesive frictional-contact model for dynamic fracture simulations under compression. International Journal of Solids and Structures 144-145, 86-99.

[3] Barenblatt, G., 1962. The mathematical theory of equilibrium cracks in brittle fracture. Advances in Applied Mechanics 7, 55-129.

[4] Benzeggagh, M. L., Kenane, M., 1996. Measurement of mixed-mode delamination fracture toughness of unidirectional glass/epoxy composites with mixed-mode bending apparatus. Compos. Sci. Technol. 56, 439-449.

[5] Borino, G., Fratini, L., Parrinello, F., 2009. Mode i failure modeling of friction stir welding joints. International Journal of Advanced Manufacturing Technology 41 (5-6), 498-503.

[6] Camanho, P., Davila, C., De Moura, M., 2003. Numerical simulation of mixed-mode progressive delamination in composite materials. J. Comp. Materials 37 (16), 1415-1438.

[7] Coleman, B., Noll, W., 1963. The thermodynamics of elastic materials with heat conduction and viscosity. Arch. Rat. Mech. Analysis 13, 167-178.

[8] Confalonieri, F., Perego, U., 2019. A new framework for the formulation and validation of cohesive mixed-mode delamination models. International Journal of Solids and Structures 164, 168-190.

[9] Crews, J., Reeder, J., 1998. A mixed-mode bending apparatus for delamination testing, technical memorandum 100662. Tech. rep., NASA Lagley.

[10] Dimitri, R., Trullo, M., Zavarise, G., De Lorenzis, L., 2014. A consistency assessment of coupled cohesive zone models for mixed-mode debonding problems. Frattura ed Integrita Strutturale 8 (29), 266-283.

[11] Dugdale, D., 1960. Yielding of steel sheets containing slits. J. Mech. Phys. Solids. 8, 100-104.

[12] Gdoutos, E., 1993. Fracture Mechanics. An introduction. Kluver Academic Publishers.

[13] Giambanco, G., La Malfa Ribolla, E., Spada, A., 2018. Meshless mesomodeling of masonry in the computational homogenization framework. Meccanica 53 (7), 1673-1697.

[14] Gulizzi, V., Rycroft, C., Benedetti, I., 2018. Modelling intergranular and transgranular micro-cracking in polycrystalline materials. Computer Methods in Applied Mechanics and Engineering 329, 168-194.

[15] Hirmand, M., Papoulia, K., 2018. A continuation method for rigid-cohesive fracture in a discontinuous galerkin finite element setting. International Journal for Numerical Methods in Engineering 115 (5), 627-650. 
[16] Högberg, J., 2006. Mixed mode cohesive law. Int. J. Fracture 141 (3-4), 549-559.

[17] Johnston, A., Davidson, B., 2014. Intrinsic coupling of near-tip matrix crack formation to mode iii delamination advance in laminated polymeric matrix composites. International Journal of Solids and Structures 51 (13), $2360-2369$.

[18] Johnston, A., Davidson, B., Simon, K., 2014. Assessment of split-beamtype tests for mode iii delamination toughness determination. International Journal of Fracture 185 (1-2), 31-48.

[19] La Malfa Ribolla, E., Rezaee Hajidehi, M., Rizzo, P., Fileccia Scimemi, G., Spada, A., Giambanco, G., 2018. Ultrasonic inspection for the detection of debonding in cfrp-reinforced concrete. Structure and Infrastructure Engineering 14 (6), 807-816.

[20] Lemaitre, J., Chaboche, J.-L., 1990. Mechanics of solids materials. Cambrige University Press.

[21] McGarry, J., O Mairtin, E., Parry, G., Beltz, G., 2014. Potential-based and non-potential-based cohesive zone formulations under mixed-mode separation and over-closure. part i: Theoretical analysis. J. Mech. Physics Solids 63 (1), 336-362.

[22] Mergheim, J., Kuhl, E., Steinmann, P., 2004. A hybrid discontinuous galerkin/interface method for the computational modelling of failure. Communications in Numerical Methods in Engineering 20 (7), 511-519.

[23] Mosler, J., Scheider, I., 2011. A thermodynamically and variationally consistent class of damage-type cohesive models. J. Mech. Physics Solids 59 (8), $1647-1668$.

[24] Park, K., Choi, H., Paulino, G., 2016. Assessment of cohesive tractionseparation relationships in abaqus: A comparative study. Mechanics Research Communications 78, 71-78.

[25] Park, K., Paulino, G., Roesler, J., 2009. A unified potential-based cohesive model of mixed-mode fracture. J. Mech. Physics Solids 57 (6), 891-908.

[26] Parrinello, F., 2013. Restraining approach for the spurious kinematic modes in hybrid equilibrium element. Computational Mechanics 52 (4), 885-901.

[27] Parrinello, F., 2018. Analytical solution of the 4enf test with interlaminar frictional effects and evaluation of mode ii delamination toughness. Journal of Engineering Mechanics 144 (4).

[28] Parrinello, F., Borino, G., 2018. Integration of finite displacement interface element in reference and current configurations. Meccanica 53 (6), 14551468 . 
[29] Parrinello, F., Failla, B., Borino, G., 2009. Cohesive-frictional interface constitutive model. Int. J. Solids Structures 46 (13), 2680-2692.

[30] Parrinello, F., Marannano, G., 2018. Cohesive delamination and frictional contact on joining surface via xfem. AIMS Materials Science 5 (1), 127-144.

[31] Parrinello, F., Marannano, G., Borino, G., 2016. A thermodynamically consistent cohesive-frictional interface model for mixed mode delamination. Engineering Fracture Mechanics 153, 61-79, cited By 0.

[32] Parrinello, F., Marannano, G., Borino, G., Pasta, A., 2013. Frictional effect in mode ii delamination: Experimental test and numerical simulation. Eng. Fract. Mech. 110, 258-269.

[33] Serpieri, R., Sacco, E., Alfano, G., 2015. A thermodynamically consistent derivation of a frictional-damage cohesive-zone model with different mode i and mode ii fracture energies. Eur. J. Mech., A/Solids 49, 13-25.

[34] Spring, D., Giraldo-Londoño, O., Paulino, G., 2016. A study on the thermodynamic consistency of the park paulino roesler (PPR) cohesive fracture model. Mechanics Research Communications 78, 100-109.

[35] Spring, D., Paulino, G., 2015. Computational homogenization of the debonding of particle reinforced composites: The role of interphases in interfaces. Computational Materials Science 109, 209-224.

[36] van den Bosch, M., Schreurs, P., Geers, M., 2006. An improved description of the exponential xu and needleman cohesive zone law for mixed-mode decohesion. Eng. Fract. Mech. 73 (9), 1220-1234.

[37] Xu, X. P., Needleman, A., 1993. Void nucleation by inclusion debonding in a crystal matrix. Modell. Simulation Mater. Sci. Eng. 2, 417-418.

[38] Zienkiewicz, O., Taylor, 2000. The Finite Element Method. 5th Edition. Butterworth-Heinemann Press. 